\title{
Inferior oblique muscle thickness in Asians
}

\author{
Yasuhiro Takahashi' \\ Hirohiko Kakizaki² \\ Takashi Nakano ${ }^{3}$ \\ Ken Asamoto ${ }^{3}$ \\ Masayoshi Iwaki \\ 'Department of Ophthalmology and \\ Visual Sciences, Osaka City University \\ Graduate School of Medicine, \\ Asahi-machi, Abeno-ku, Osaka, Japan; \\ 2Department of Ophthalmology; \\ ${ }^{3}$ Anatomy, Aichi Medical University, \\ Nagakute, Aichi, Japan
}

\begin{abstract}
The purpose of this study was to examine the inferior oblique muscle (IOM) thickness in Asians by the quasi-sagittal plane of the orbits. Specimens were examined from 23 orbits of 18 Asians ( 9 orbits in males, 14 in females), aged 63 to 97 years at death. All specimens were fixed in $10 \%$ buffered formalin before use. The orbital contents were incised parallel to the optic nerve, after which the lengths of both short and long axes of the IOM were measured and the ratio of short to long axes was calculated. The mean lengths of each short or long axis of the IOM were $2.27 \pm 0.49 \mathrm{~mm}$ and $7.23 \pm 0.97 \mathrm{~mm}$, respectively. The average ratio was $0.32 \pm$ 0.07 . There were no significant differences in laterality or gender. This study determined the normal IOM thickness in Asian cadavers, the outcome of which could be useful for detection of IOM involvement in Graves' orbitopathy.
\end{abstract}

Keywords: inferior oblique muscle, Asian, thickness, quasi-sagittal plane

Inferior oblique muscle (IOM) involvement has been recently highlighted in Graves' orbitopathy (Nishida et al 2001; Kakizaki 2007; Kakizaki et al 2007). Previously, the IOM has been excluded from studies of the extraocular muscles in this condition (Demer and Kerman 1994; Lennerstrand et al 2007; Sheikh et al 2007). Therefore, there is little information regarding IOM involvement in Graves' orbitopathy.

Thickness of the IOM is clinically more useful than the cross-sectional area for evaluation of orbital involvement because of its simple numeric expression. To date, the cross-sectional area of the IOM has been reported with magnetic resonance imaging (MRI) in the quasi-sagittal plane (Demer et al 2003; Detorakis et al 2003; Kono and Demer 2003) and with cadavers in the coronal plane (Nakagawa 1965), but these measurements are not practical for use in a daily examination.

The quasi-sagittal plane (parallel to the long axis of the orbit) has been recently thought to be a reliable plane for morphological studies of the IOM (Kono and Demer 2003).

Although only one study has reported the thickness of the IOM, the images were taken with a coronal view of the MRI (Bourlet et al 1998). Since the coronal plane is not perpendicular to the long axis of the IOM, its size might be inaccurately estimated (Wing et al 1979; Demer and Kerman 1994).

In the present study, we examined normal IOM thickness in Asian cadavers in the quasi-sagittal plane of the orbits.

\section{Materials and methods}

Twenty-three post mortem orbits (12 right, 11 left) were examined in 18 Asians, aged from 63 to 97 years at death (mean age: 77.9 years). Nine orbits of 8 cadavers were from males, and 14 orbits of 10 cadavers were from females. The orbits with globe enucleation were excluded from the study (Detorakis et al 2003). None of the cadavers had any clinical evidence of Graves' disease, cerebral nerve palsy, strabismus, or any orbital diseases (Ossoinig and Hermsen 1983; Kono and Demer 2003). All specimens were fixed with 10\% buffered formalin before use. Consent and approval to use all cadavers for educational 
purposes and studies was obtained and all cadavers were registered in the cadaveric service of Aichi Medical University. All methods for securing human tissue were humane, and complied with the tenets of the Declaration of Helsinki.

We removed the orbits using a binocular loupe (high resolution prismatic $[\mathrm{HRP}] \times 3.5,420 \mathrm{~mm} / 16$ inches, Heine, Herrsching, Germany). After conducting a full thickness incision of the periosteum around the complete circumference of the orbits, the periosteum was detached near the orbital apex. We cut the nerves, blood vessels, and the nasolacrimal duct out of the orbital wall. The lateral orbital wall was then removed about $3 \mathrm{~cm}$ from the orbital rim and retrobulbar content was incised with a sharp scalpel parallel to the coronal section. We incised the removed orbital contents parallel to the optic nerve, and then measured the lengths of both the short and long axes of the IOM (Figure 1A and B).

The ratio of short to long axes was also calculated (Aydin et al 2003). Laterality and difference in gender were statistically analyzed using a Mann-Whitney U-test. Statistical significance was defined as $\mathrm{p}<0.05$. All statistical analysis was carried out using SPSS II for Windows (SPSS Japan Inc., Tokyo, Japan).

\section{Results}

The mean length of each short or long axis of the IOM ( \pm standard deviation), and the ratio of short to long axes are given in Table 1. There was no significant difference in laterality for the short or long axes (short: $p=0.559$, long: $p=0.255$ ). There was also no significant difference in laterality for the average ratio $(\mathrm{p}=0.424)$. There were no significant differences between sexes for the short or long axis (Table 1, short axis: $\mathrm{p}=0.083$, long axis: $\mathrm{p}=0.083$ ). There was also no significant difference in the mean ratio between sexes $(p=0.900)$.

\section{Discussion}

We have described the normal IOM thickness in the quasisagittal plane in Asian cadavers. Muscle thickness can be practically used for quantitative evaluation of the muscle cross-section and can be easily compared with the optic nerve diameter as a reference (Nishikawa et al 1993). Although the use of muscle cross-sectional area or volume is sometimes recommended to evaluate muscle size (Szucs-Farkas et al 2002; Majos et al 2007), this method is time-consuming and needs an additional special software (Aydin et al 2003).

The shape of the extraocular muscle in cross-section is a thin ellipse, in which the IOM is the thinnest (Aydin et al 2003). In Graves' orbitopathy, extraocular muscles enlarge more along their short axis than the long axis, which means
A

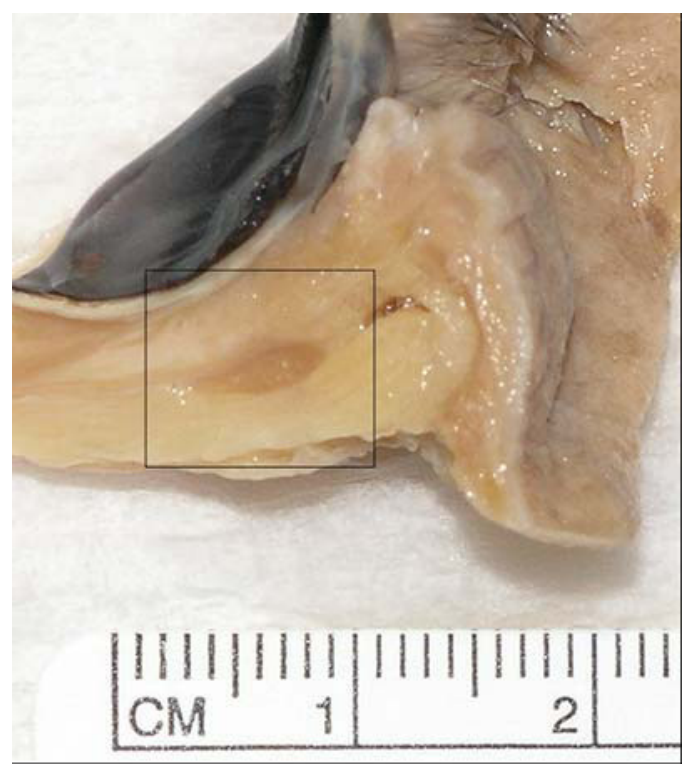

B

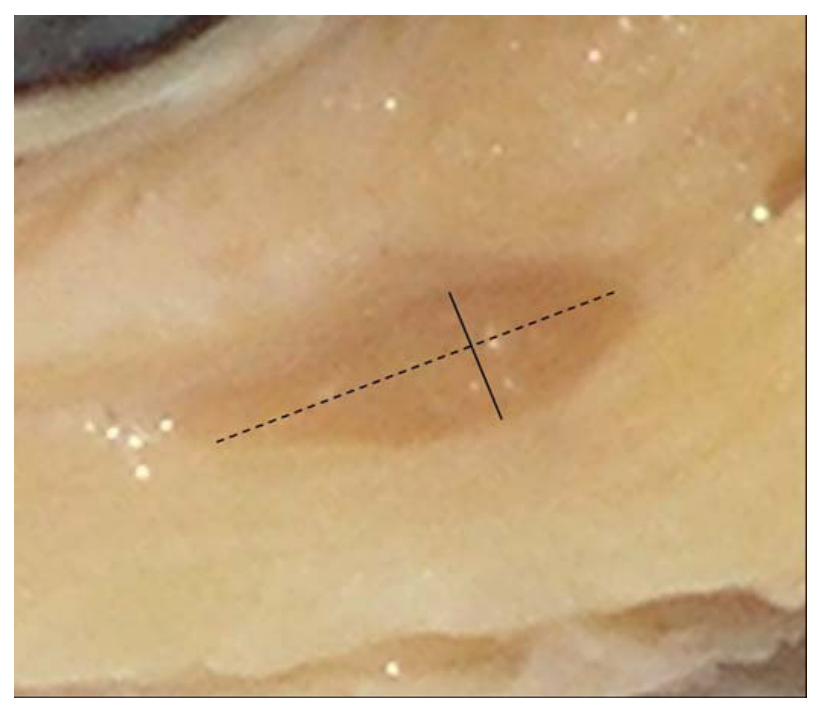

Figure I Photograph of a quasi-sagittal section of the cadaveric orbit. A.Whole aspect. B. Magnification of the square in A. The maximum lengths of both short (solid line) and long (dashed line) axes were measured.

that the shape of the cross-section is a thicker ellipse (Aydin et al 2003). In this regard, the thickness of extraocular muscles can be a useful indicator to detect an involvement of Graves' orbitopathy and the morbidly thickened IOM can be grasped more easily than the other muscles.

The lengths of both short and long axes in males were slightly larger than those in females, although this was not statistically significant. The relationship between muscle size and gender has been previously reported (Ozgen and Ariyurek 1998; Ozgen and Aydingoz 2000; Aydin et al 2003). It was shown that the thickness of the superior oblique muscle 
Table I Mean lengths and the ratio of short to long axes ( $\mathrm{mm} \pm$ standard deviation) of inferior oblique muscles in Asians and comparison of each value regarding laterality or sex

\begin{tabular}{|c|c|c|c|c|c|}
\hline & All $(n=23)$ & Right (n = I2) & Left $(n=I I)$ & Male $(n=9)$ & Female $(n=14)$ \\
\hline Age & 77.9 & 79.5 & 77.9 & 76.6 & 79.4 \\
\hline Short axis & $2.27 \pm 0.49$ & $2.18 \pm 0.42$ & $2.37 \pm 0.56$ & $2.49 \pm 0.52$ & $2.12 \pm 0.43$ \\
\hline Long axis & $7.23 \pm 0.97$ & $6.98 \pm 0.92$ & $7.50 \pm 0.99$ & $7.58 \pm 0.85$ & $7.01 \pm 1.01$ \\
\hline Ratio & $0.32 \pm 0.07$ & $0.32 \pm 0.07$ & $0.32 \pm 0.06$ & $0.33 \pm 0.08$ & $0.31 \pm 0.06$ \\
\hline
\end{tabular}

in males was greater than that in females (Ozgen and Aydingoz 2000). Since the IOM is the antagonist of the superior oblique muscle, males may have a thicker IOM than females in order to maintain a functional balance between the two muscles.

MRI has been developed as a useful method for anatomical studies because of its high resolution (Boonstra et al 1983; Tian et al 2000). However, since the IOM is situated in a crowded area with the inferior rectus muscle and the globe (Nakagawa 1965), it is sometimes difficult to judge definite IOM diameters in the quasi-sagittal plane even with highresolution MRI (Tian et al 2000). Once the correct thickness of the IOM is determined in cadavers, it can be appropriately applied to the MRI images as a reliable reference.

Approximately $15 \%$ of nonmuscular tissue shrinks in the process of formalin fixation, and accordingly autopsy measurements sometimes differ from those in vivo (Boonstra et al 1983). However, IOM thickness in this study was similar to that in a former MRI study, although statistical significance was not shown (Bourlet et al 1998) $(p=0.162$, Mann-Whitney U-test). Therefore, muscle may not be affected by formalin fixation. The purpose of the study was to determine a more accurate measure of IOM thickness in the quasi-sagittal plane than that determined by MRI in the coronal plane. Since MRI is only an image, and not a true figure, we needed to determine the true diameter using cadavers to fully understand the MRI findings.

Muscle diameter tends to increase with age until 40 to 60 years-old, but after that the diameter decreases, although no statistical difference has been shown (Lee et al 2001). Several factors affect muscle enlargement, such as metabolic factors, hormonal changes, neuronal influences and development of the motor visual system (Sacca et al 2000). In our study, the cadavers' average age at death was 77.9 years and all of them were older than 60 years. Therefore, the present measurements might be a little smaller than those expected from 40 to 50 years of age. However, as the difference is very small (Lee et al 2001), the present data can be applied to each patient, as well as in those in the 40 to 50 year age range, in which Graves' orbitopathy commonly occurs (Rubin and Sadun 2004).
In conclusion, we have described the normal IOM thickness in Asian cadavers. Our findings could be useful for detection of IOM involvement in Graves' orbitopathy.

\section{Disclosure}

The authors have no financial support and no financial interest related to this manuscript.

\section{References}

Aydin K, Güven K, Sencer S, et al. 2003. A new MRI method for the quantitative evaluation of extraocular muscle size in thyroid ophthalmopathy. Neuroradiol, 45:184-7.

Boonstra H, Oosterhuis JW, Oosterhuis AM, et al. 1983. Cervical tissue shrinkage by formaldehyde fixation, paraffin wax embedding, section cutting and mounting. Virchows Arch, 402:195-201.

Bourlet P, Carrie D, Garcier JM, et al. 1998. Study of the inferior oblique muscle of the eye by MRI. Surg Radiol Anat, 20:199-21.

Demer JL, Kerman BM. 1994. Comparison of standardized echography with magnetic resonance imaging to measure extraocular muscle size. Am J Ophthalmol, 118:351-61.

Demer JL, Oh SY, Clark RA, et al. 2003. Evidence for a pulley of the inferior oblique muscle. Invest Ophthalmol Vis Sci, 44:3856-65.

Detorakis ET, Engstrom RE, Straatsma BR, et al. 2003. Functional anatomy of the anophthalmic socket: insights from magnetic resonance imaging. Invest Ophthalmol Vis Sci, 44:4307-13.

Kakizaki H. 2007. Inflammatory swelling of the inferior oblique muscle in thyroid associated ophthalmopathy. Clin Ophthalmol, $1: 189-92$.

Kakizaki H, Zako M, Iwaki M. 2007. Thyroid associated inferior oblique myopathy. Ophthalmology, 114:2106.

Kono R, Demer JL. 2003. Magnetic resonance imaging of the functional anatomy of the inferior oblique muscle in superior oblique palsy. Ophthalmology, 110:1219-29.

Lee JS, Lim DW, Lee SH, et al. 2001. Normative measurements of Korean orbital structures revealed by computerized tomography. Acta Ophthalmol Scand, 79:197-200.

Lennerstrand G, Tian S, Isberg B, et al. 2007. Magnetic resonance imaging and ultrasound measurements of extraocular muscles in thyroid-associated ophthalmopathy at different stages of the disease. Acta Ophthalmol Scand, 85:192-201.

Majos A, Grzelak P, Mlynarczyk W, et al. 2007. Eyeball mucles' diameter versus volume estimated by numerical image segmentation. Eur $J$ Ophthalmol, 17:287-93.

Nakagawa T. 1965. [Topographic anatomical studies on the orbit and its contents]. Nippon Ganka Gakkai Zasshi, 69:2155-9.

Nishida Y, Tian S, Isberg B, et al. 2001. MRI measurements of orbital tissues in dysthyroid ophthalmopathy. Graefes Arch Clin Exp Ophthalmol, 239:824-31.

Nishikawa M, Yoshimura M, Toyoda N, et al. 1993. Correlation of orbital muscle changes evaluated by magnetic resonance imaging and thyroidstimulating antibody in patients with Graves' ophthalmopathy. Acta Endocrinol, 129:213-19. 
Ossoinig KC, Hermsen VM. 1983. Myositis of extraocular muscles diagnosed with standardized echography. Doc Opthalmol Proc Ser, 38:381-9.

Ozgen A, Ariyurek M. 1998. Normative measurement of orbital structure using CT. Am J Roentgenol, 170:1093-6.

Ozgen A, Aydingoz U. 2000. Normative measurements of orbital structures using MRI. J Comput Assist Tomogr, 24:493-6.

Rubin RM, Sadun AA. 2004. Graves' dysthyroid ophthalmopathy. In: Yanoff M, Duker JS (ed). Ophthalmology, 2nd ed. St. Louis: Mosby, pp. 1345-8.

Sacca S, Polizzi A, Macri A, et al. 2000. Echographic study of extraocular muscle thickness in children and adults. Eye, 14:765-9.
Sheikh M, Abalkhail S, Doi SAR, et al. 2007. Normal measurement of orbital structures: implications for the assessment of Graves' ophthalmopathy. Australas Radiol, 51:253-6.

Szucs-Farkas Z, Toth J, Balazs E, et al. 2002. Using morphologic parameters of extraocular muscles for diagnosis and follow-up of Graves' ophthalmopathy: diameters, areas, or volumes? Am J Radiol, 179:1005-10.

Tian S, Nishida Y, Isberg B, et al. 2000. MRI measurements of normal extraocular muscles and other orbital structures. Graefes Arch Clin Exp Ophthalmol, 238:393-404.

Wing SD, Hunsaker JN, Anderson RE, et al. 1979. Direct sagittal computed tomography in Graves' ophthalmopathy. J Comput Assist Tomogr, $3: 820-4$. 